\title{
DIFFERENTIAL INCLUSIONS FOR DIFFERENTIAL FORMS
}

\author{
SAUGATA BANDYOPADHYAY \\ Section de Mathématiques, EPFL \\ 1015 Lausanne, Switzerland \\ email: saugata.bandyopadhyay@epfl.ch \\ Ana Cristina Barroso \\ CMAF, Universidade de Lisboa \\ Av. Prof. Gama Pinto, 2 \\ 1649-003 Lisboa, Portugal \\ and \\ Departamento de Matemática \\ Faculdade de Ciências \\ Universidade de Lisboa \\ 1749-016 Lisboa, Portugal \\ email:abarroso@ptmat.fc.ul.pt \\ Bernard Dacorogna \\ Section de Mathématiques, EPFL \\ 1015 Lausanne, Switzerland \\ email: bernard.dacorogna@epfl.ch

\section{José Matias} \\ Departamento de Matemática \\ Instituto Superior Técnico \\ Av. Rovisco Pais, 1 \\ 1049-001 Lisboa, Portugal \\ email:jmatias@math.ist.utl.pt
}

\begin{abstract}
We study necessary and sufficient conditions for the existence of solutions in $W_{0}^{1, \infty}\left(\Omega ; \Lambda^{k}\left(\mathbb{R}^{n}\right)\right)$ of the problem

$$
d \omega(x) \in E, \text { a.e. in } \Omega
$$

where $E \subseteq \Lambda^{k+1}\left(\mathbb{R}^{n}\right)$ is a given set. Special attention is given to the case of the curl (i.e. $k=1$ ), particularly in dimension 3. Some applications to the calculus of variations are also stated.
\end{abstract}




\section{Introduction}

In this paper we search for solutions $\omega \in W_{0}^{1, \infty}\left(\Omega ; \Lambda^{k}\left(\mathbb{R}^{n}\right)\right)$ of the differential inclusion

$$
(P) \quad d \omega(x) \in E \text {, a.e. in } \Omega
$$

where:

- $\Omega \subset \mathbb{R}^{n}$ is a bounded, open set,

- $\omega \in \Lambda^{k}\left(\mathbb{R}^{n}\right)$ is a $k$-form, $0 \leq k \leq n-1$ (we will in fact identify, by abuse

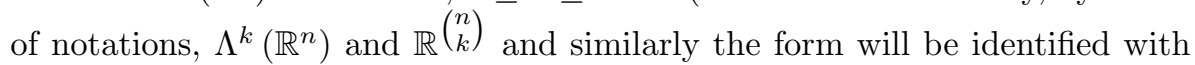
its components, i.e. $\left.\omega: \Omega \subset \mathbb{R}^{n} \rightarrow \mathbb{R}^{\left(\begin{array}{l}n \\ k\end{array}\right)}\right)$,

- $d \omega \in \Lambda^{k+1}\left(\mathbb{R}^{n}\right)$ is the exterior derivative and is, as above, seen as a vector field $d \omega: \Omega \subset \mathbb{R}^{n} \rightarrow \mathbb{R}^{\left(\begin{array}{c}n \\ k+1\end{array}\right)}$,

- $E \subseteq \Lambda^{k+1}\left(\mathbb{R}^{n}\right)$ is a given set,

- $W_{0}^{1, \infty}\left(\Omega ; \Lambda^{k}\left(\mathbb{R}^{n}\right)\right)$ stands for the Sobolev space of functions so that $\omega \in$ $L^{\infty}\left(\Omega ; \Lambda^{k}\left(\mathbb{R}^{n}\right)\right), \frac{\partial \omega}{\partial x_{j}} \in L^{\infty}\left(\Omega ; \Lambda^{k}\left(\mathbb{R}^{n}\right)\right)$ for all $j=1, \ldots, n$ and $\omega=0$ on $\partial \Omega$.

Our goal is to identify necessary and sufficient conditions for the existence of solutions of problem $(P)$.

The most important case is, of course, the one where $k=0$ (we therefore have, with our convention, that $d \omega=\operatorname{grad} \omega)$. It has received considerable attention, in particular, by Bressan-Flores [4], Cellina [5], [6], DacorognaMarcellini [10] and Friesecke [12]. We refer, for a survey as well as for an extensive bibliography, to Dacorogna [7].

The next important case is the one where $k=1$ (especially when $n=3$ ) which, with our convention, yields $d \omega=\operatorname{curl} \omega$. This is a particularly important problem for applications, such as the Ginzburg-Landau model of ferromagnetism, and we refer to Barroso-Matias [3], Dacorogna-Fonseca [9], DeSimoneDolzmann [11], James-Kinderlehrer [17] and to the references therein.

Another interesting, and particularly simple case is $k=n-1$ where $d \omega=$ $\operatorname{div} \omega$. It has been considered by Barroso-Matias in [3].

Our purpose in this article is to unify the study of the different cases mentioned above (gradient, curl and divergence cases) as well as to deal with all the intermediate cases $(2 \leq k \leq n-2)$.

In section 3 we discuss some necessary and some sufficient conditions for the existence of solutions of problem $(P)$ in the case of a general $k$-form. For the sufficiency part the idea is to find solutions of the form $\omega(x)=u(x) b$, for an appropriate non-trivial $k$-form $b$. The problem is then reduced to a gradient problem.

In Section 4 the previously obtained necessary and sufficient conditions are improved for the case $k=1$. The improvement is particularly significant for 
the necessary condition. In the last part of this section we consider the case $k=1$ and $n=3$. As for the classical case $k=0$, in this setting we show that the necessary and sufficient conditions are exactly the same. Also, the proof of the necessary condition can be greatly simplified. We obtain the following result (cf. Theorem 4.15):

Theorem 1.1 Let $\Omega \subset \mathbb{R}^{3}$ be a bounded, open set and let $E \subset \mathbb{R}^{3}$ be such that $0 \notin E$. The following two properties are equivalent:

(i) There exists $\omega \in W_{0}^{1, \infty}\left(\Omega ; \mathbb{R}^{3}\right)$ with $\operatorname{curl} \omega \in E$ a.e. in $\Omega$.

(ii) There exists a subset $F \subseteq E$ such that $\operatorname{dim} \operatorname{span} F \geq 2$ and $0 \in \operatorname{ri}(\operatorname{co} F)$ (the relative interior of the convex hull of $F$ ).

This result improves Barroso-Matias [3], Dacorogna-Fonseca [9] and JamesKinderlehrer [17], see Remark 4.16 for details.

In the case of the gradient it is known that there exists $\omega \in W_{0}^{1, \infty}(\Omega)$ with $\operatorname{grad} \omega \in E$ a.e. in $\Omega$ if and only if $0 \in E \cup$ int co $E$. We therefore see that we have more freedom for the curl than for the gradient.

The existence results for the differential inclusion problem can be applied, following the ideas in Cellina [5], [6] and Friesecke [12], to obtain existence of solutions for a non-convex variational problem. In particular one can show that:

Theorem 1.2 Let $\Omega \subset \mathbb{R}^{n}$ be a bounded, open set, $0 \leq k \leq n-1$ and

$$
f: \Lambda^{k+1}\left(\mathbb{R}^{n}\right) \longrightarrow \mathbb{R}_{+}
$$

be lower semicontinuous. Let

$$
\text { (Q) } \quad \inf \left\{\int_{\Omega} f(d \omega(x)) d x: \omega \in W_{0}^{1, \infty}\left(\Omega ; \Lambda^{k}\left(\mathbb{R}^{n}\right)\right)\right\}
$$

and

$$
K=\left\{\xi \in \Lambda^{k+1}\left(\mathbb{R}^{n}\right): f^{* *}(\xi)<f(\xi)\right\},
$$

where $f^{* *}$ is the convex envelope of $f$. Assume that $K$ is connected and $0 \in K$. If $K$ is bounded and $f^{* *}$ is affine on $K$ then $(Q)$ has a solution.

More details and variants on this problem can be found in [1] and [2].

We conclude with the trivial observation that we can replace the condition $\omega=0$ on $\partial \Omega$ by any affine boundary data or, more generally, by any boundary data $\varphi$ such that $d \varphi$ is constant, with no changes in the proofs. 


\section{Notations}

We start with some notations which are used throughout this paper. Although these notations are somewhat standard we mention them here for the sake of completeness. For more details on exterior algebra we refer to [13], for analysis of differential forms see [8], [15] and [16] and for convex analysis we refer to $[14]$ and $[18]$.

1. $\mathbb{R}_{+}$denotes the set of all non-negative real numbers.

2. Let $V$ be an $n$-dimensional inner product space over $\mathbb{R}$.

- For $E \subseteq V, E \neq \emptyset$, we write span $E$ to denote the subspace spanned by $E$.

- Let $W$ be a subspace of $V$. We write $\operatorname{dim} W$ to denote the dimension of $W$.

- For each $k \in \mathbb{N}$, we write $\Lambda^{k}(V)$ to denote the vector space of all alternating $k$-linear maps $\varphi: \underbrace{V \times \cdots \times V}_{k-\text { times }} \rightarrow \mathbb{R}$. For $k=0$, we set $\Lambda^{0}(V)=\mathbb{R}$. Note that $\Lambda^{k}(V)=\{0\}$ for $k>n$ and $\operatorname{dim} \Lambda^{k}(V)=\left(\begin{array}{l}n \\ k\end{array}\right)$ for $0 \leqslant k \leqslant n$.

- $\wedge$ denotes the usual wedge product.

- If $\left\{e_{j} \mid j=1, \ldots, n\right\}$ is a basis of $V$, then $\left\{e_{j_{1}} \wedge \ldots \wedge e_{j_{k}} \mid 1 \leqslant j_{1}<\right.$ $\left.\ldots<j_{k} \leqslant n\right\}$ is a basis of $\Lambda^{k}(V)$.

- For $x \in V \backslash\{0\}, \pi_{x}: V \rightarrow V / \mathbb{R} x$ denotes the standard projection map which we will call canonical epimorphism.

- Let $U$ be a real vector space and let $T: V \rightarrow U$ be a linear transformation. Then there exists a unique linear transformation $T^{\star}: \Lambda^{2}(V) \rightarrow \Lambda^{2}(U)$ such that

$$
T^{\star}\left(v_{1} \wedge v_{2}\right)=T\left(v_{1}\right) \wedge T\left(v_{2}\right) \text { for all } v_{1}, v_{2} \in V .
$$

We say that $T^{\star}$ is induced by $T$. In this paper, we will consider the epimorphism $\pi_{x}^{\star}: \Lambda^{2}(V) \rightarrow \Lambda^{2}(V / \mathbb{R} x)$ induced by $\pi_{x}: V \rightarrow V / \mathbb{R} x$, where $x \in V \backslash\{0\}$. The map $\pi_{x}^{\star}$ has some important properties:

(a) $\pi_{x}^{\star}$ is surjective, which accounts for the word epimorphism,

(b) $\operatorname{ker} \pi_{x}^{\star}=x \wedge V$.

- Let $W$ be a subspace of $\Lambda^{2}(V)$ and let $x \in V$. We write

$$
\begin{aligned}
& W(x)=\{w \in W \mid w \wedge x=0\} \\
& W_{x}^{\star}=\pi_{x}^{\star}(W) .
\end{aligned}
$$


- For $b \in \Lambda^{k}(V)$, we write

$$
V_{b}=\{x \wedge b \mid x \in V\} \subset \Lambda^{k+1}(V) .
$$

Note that $\operatorname{dim} V_{b}=n-k$ if $b \in V \backslash\{0\}$.

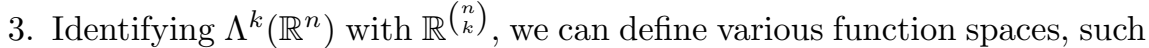
as $C^{1}\left(\Omega ; \Lambda^{k}\left(\mathbb{R}^{n}\right)\right), W^{1, p}\left(\Omega ; \Lambda^{k}\left(\mathbb{R}^{n}\right)\right)$ and $W_{0}^{1, p}\left(\Omega ; \Lambda^{k}\left(\mathbb{R}^{n}\right)\right), 1 \leqslant p \leqslant \infty$, in a straightforward way.

4. If $\omega \in W^{1, p}\left(\Omega ; \Lambda^{k}\left(\mathbb{R}^{n}\right)\right)$, the exterior derivative $d \omega$ belongs to $L^{p}\left(\Omega ; \Lambda^{k+1}\left(\mathbb{R}^{n}\right)\right)$ and it is defined by

$$
(d \omega)_{i_{1}, \ldots, i_{k+1}}=\sum_{\gamma=1}^{k+1}(-1)^{\gamma-1} \frac{\partial \omega_{i_{1} \ldots i_{\gamma-1} i_{\gamma+1} \ldots i_{k+1}}}{\partial x_{i_{\gamma}}},
$$

for $1 \leq i_{1}<\ldots<i_{k+1} \leq n$. In particular, if $k=0$ then $d \omega \simeq \operatorname{grad} \omega$ and if $k=n, d \omega=0$. If $k=1$, for $1 \leq i<j \leq n$

$$
(d \omega)_{i j}=\frac{\partial \omega_{j}}{\partial x_{i}}-\frac{\partial \omega_{i}}{\partial x_{j}}
$$

i.e. $d \omega \simeq \operatorname{curl} \omega$. Finally, if $k=n-1$ then $d \omega \simeq \operatorname{div} \omega$.

5. $\mathcal{H}^{k}$ denotes the $k$-dimensional Hausdorff measure.

6. co $C$ denotes the convex hull of $C \subseteq \mathbb{R}^{n}$ and $\overline{\operatorname{co}} C$ its closure.

7. For a convex set $C \subseteq \mathbb{R}^{n}$,

(a) $\operatorname{Aff}(C)$ denotes the affine hull of $C$ which is the intersection of all affine subsets of $\mathbb{R}^{n}$ containing $C$.

(b) $\operatorname{ri}(C)$ denotes the relative interior of $C$ which is the interior of $\mathrm{C}$ with respect to the topology relative to the affine hull of $C$.

(c) $\operatorname{rbd}(C)$ denotes the relative boundary of $C$ which is $\bar{C} \backslash \operatorname{ri}(C)$.

\section{The case of a $k$-form}

In this section we obtain necessary and sufficient conditions for the existence of solutions to the differential inclusion problem

$$
d \omega \in E, \text { a.e. in } \Omega
$$

where $\omega$ is a general $k$-form $(0 \leq k \leq n-1)$ and $E \subseteq \Lambda^{k+1}\left(\mathbb{R}^{n}\right)$ is a given set. We begin with the following definition. 
Definition 3.1 An element $b \in \Lambda^{k}\left(\mathbb{R}^{n}\right) \backslash\{0\}$ is said to be decomposable if it is possible to write $b=\stackrel{\wedge}{i=1}_{\wedge} b^{i}$ for some vectors $b^{1}, \ldots, b^{k} \in \mathbb{R}^{n}$.

The main theorem of this section is

Theorem 3.2 [Sufficient condition] Let $\Omega \subset \mathbb{R}^{n}$ be open and bounded. For $1 \leqslant k \leqslant n-1$, let $b \in \Lambda^{k}\left(\mathbb{R}^{n}\right) \backslash\{0\}$ be decomposable. Let $E \subset V_{b} \subset \Lambda^{k+1}\left(\mathbb{R}^{n}\right)$ be such that

$$
0 \in E \cup \operatorname{ri}(\operatorname{co} E) \text { and } \operatorname{dim} \operatorname{span} E=n-k .
$$

Then there exists $\omega \in W_{0}^{1, \infty}\left(\Omega ; \Lambda^{k}\left(\mathbb{R}^{n}\right)\right)$ such that

$$
d \omega \in E \text {, a.e. in } \Omega \text {. }
$$

Moreover, $\omega$ is of the form

$$
\omega(x)=u(x) b
$$

for some $u \in W_{0}^{1, \infty}(\Omega)$.

Remark 3.3 (i) In fact the theorem is also valid for $k=0$ and is well known (cf. [6], [10], [12]). It reads as: if $0 \in E \cup \operatorname{int} \operatorname{co} E$ then there exists $\omega \in W_{0}^{1, \infty}(\Omega)$ such that

$$
d \omega=\operatorname{grad} \omega \in E \text {, a.e. in } \Omega .
$$

(ii) For the case $k=1$ see Theorem 4.13; if, in addition, $n=3$ the theorem can be improved and takes the simpler form given in Theorem 4.15.

Before we embark on the proof of the theorem we need a couple of lemmas which we will not prove here. For the proof of these two lemmas we refer to [6], Lemma 2.11 of [10] and [12].

Lemma 3.4 Let $E \subset \mathbb{R}^{N}$ with $\operatorname{dim} \operatorname{span} E=n \leqslant N$ and let $0 \in \operatorname{ri}(\operatorname{co} E)$. Then there exist $m \geqslant n+1, z^{\alpha} \in E, t^{\alpha}>0$ such that

$$
\sum_{\alpha=1}^{m} t^{\alpha} z^{\alpha}=0, \sum_{\alpha=1}^{m} t^{\alpha}=1, \operatorname{dim} \operatorname{span}\left\{z^{\alpha} \mid \alpha=1, \ldots, m\right\}=n .
$$

Lemma 3.5 (Pyramids) Let $\Omega \subset \mathbb{R}^{n}$ be open and bounded. Let $z^{\alpha} \in \mathbb{R}^{n}$, $t^{\alpha}>0, \alpha=1, \ldots, m$, with $m \geq n+1$, be such that

$$
\sum_{\alpha=1}^{m} t^{\alpha} z^{\alpha}=0, \sum_{\alpha=1}^{m} t^{\alpha}=1, \operatorname{dim} \operatorname{span}\left\{z^{\alpha} \mid \alpha=1, \ldots, m\right\}=n .
$$

Then there exists $u \in W_{0}^{1, \infty}(\Omega)$ satisfying

$$
\operatorname{grad} u \in\left\{z^{\alpha} \mid \alpha=1, \ldots, m\right\} \text {, a.e. in } \Omega \text {. }
$$


We now proceed with the proof of the theorem.

Proof. If $0 \in E$, the proof is trivial. We simply take $\omega=d \varphi$, for any $\varphi \in$ $W_{0}^{2, \infty}\left(\Omega ; \Lambda^{k-1}\left(\mathbb{R}^{n}\right)\right)$. Hence, we assume that $0 \notin E$ and $0 \in \operatorname{ri}(\operatorname{co} E)$.

Since $b \in \Lambda^{k}\left(\mathbb{R}^{n}\right) \backslash\{0\}$ is decomposable there exist $k$ linearly independent vectors $b^{1}, \ldots, b^{k} \in \mathbb{R}^{n}$ such that $b=\stackrel{\wedge}{i=1}^{k} b^{i}$.

We divide the proof in two cases.

Case 1: $b^{1}, \ldots, b^{k}$ are orthonormal.

Step 1. Since the set $S=\left\{b^{j} \mid j=1, \ldots, k\right\}$ is orthonormal, we can extend $S$ to an orthonormal basis $\left\{b^{j} \mid j=1, \ldots, n\right\}$ of $\mathbb{R}^{n}$. Let $R \in O(n)$ be such that $R e^{j}=b^{j}$, for all $j=1, \ldots, n$. As $\operatorname{dim} \operatorname{span} E=n-k$, Lemma 3.4 yields $z^{\alpha} \in E, t^{\alpha}>0, \alpha=1, \ldots, m$, such that

$$
\sum_{\alpha=1}^{m} t^{\alpha} z^{\alpha}=0, \sum_{\alpha=1}^{m} t^{\alpha}=1, \operatorname{dim} \operatorname{span}\left\{z^{\alpha} \mid \alpha=1, \ldots, m\right\}=n-k
$$

for some $m \geqslant n-k+1$. Also, since $E \subset V_{b}$, there exists $a^{\alpha} \in \mathbb{R}^{n}$, for all $\alpha=1, \ldots, m$, such that

$$
\begin{array}{cl}
\text { i) } & z^{\alpha}=a^{\alpha} \wedge b \\
\text { ii) } & a^{\alpha} \in \operatorname{span}\left\{b^{j} \mid j=1, \ldots, k\right\}^{\perp} \text { and } \\
\text { iii) } & \operatorname{dim} \operatorname{span}\left\{a^{\alpha} \mid \alpha=1, \ldots, m\right\}=n-k .
\end{array}
$$

Note that $a^{\alpha}$-s satisfying $i$ ) and $i$ ) automatically satisfy $\left.i i i\right)$ because $\operatorname{dim} \operatorname{span}\left\{z^{\alpha} \mid \alpha=1, \ldots, m\right\}=n-k$.

Step 2. We apply Lemma 3.5 to the vectors $\xi^{\alpha, \beta} \in \mathbb{R}^{n}, \alpha=1, \ldots, m$ and $\beta=1, \ldots, 2^{k}$, given by

$$
\xi^{\alpha, 1}=\left(\begin{array}{c}
1 \\
\vdots \\
1 \\
1 \\
\widetilde{a}_{k+1}^{\alpha} \\
\vdots \\
\widetilde{a}_{n}^{\alpha}
\end{array}\right), \xi^{\alpha, 2}=\left(\begin{array}{c}
1 \\
\vdots \\
1 \\
-1 \\
\widetilde{a}_{k+1}^{\alpha} \\
\vdots \\
\widetilde{a}_{n}^{\alpha}
\end{array}\right), \ldots, \xi^{\alpha, 2^{k}}=\left(\begin{array}{c}
-1 \\
\vdots \\
-1 \\
-1 \\
\widetilde{a}_{k+1}^{\alpha} \\
\vdots \\
\widetilde{a}_{n}^{\alpha}
\end{array}\right)
$$


where $\widetilde{a}^{\alpha}=R^{T} a^{\alpha}$ for all $\alpha=1, \ldots, m$, to obtain $u \in W_{0}^{1, \infty}\left(R^{T} \Omega\right)$ such that

$$
\operatorname{grad} u(y) \in\left\{\left(\begin{array}{c} 
\pm 1 \\
\vdots \\
\pm 1 \\
\widetilde{a}_{k+1}^{\alpha} \\
\vdots \\
\widetilde{a}_{n}^{\alpha}
\end{array}\right)\right\} \text {, for a.e. } y \in R^{T} \Omega
$$
hold:

The hypotheses of the lemma are satisfied since the following conditions

$$
\begin{array}{ll}
\left.i^{\prime}\right) & 2^{k} m \geqslant n+1 \\
\left.i i^{\prime}\right) \quad & \operatorname{dim} \operatorname{span}\left\{\xi^{\alpha, \beta} \in \mathbb{R}^{n} \mid \alpha=1, \ldots, m ; \beta=1, \ldots, 2^{k}\right\}=n \\
\left.i i i^{\prime}\right) & \sum_{\alpha=1}^{m} \sum_{\beta=1}^{2^{k}} \tau^{\alpha, \beta} \xi^{\alpha, \beta}=0 \\
\left.i v^{\prime}\right) & \sum_{\alpha=1}^{m} \sum_{\beta=1}^{2^{k}} \tau^{\alpha, \beta}=1
\end{array}
$$

where $\tau^{\alpha, \beta}=\frac{t^{\alpha}}{2^{k}}$, for all $\alpha=1, \ldots, m$ and $\beta=1, \ldots, 2^{k}$.

Conditions $i^{\prime}$ ) and $\left.i v^{\prime}\right)$ are trivial to check so we prove only $i i^{\prime}$ ) and $i i i^{\prime}$ ).

Proof of ii'): Let $x \in \mathbb{R}^{n}$ be such that $\left\langle x ; \xi^{\alpha, \beta}\right\rangle=0$, for all $\alpha=1, \ldots, m$, $\beta=1, \ldots, 2^{k}$. We show that $x=0$.

For each $r, 1 \leqslant r \leqslant k$, we choose $1 \leqslant \beta, \gamma \leqslant 2^{k}$ such that

$$
\xi^{\alpha, \beta}-\xi^{\alpha, \gamma}=2 e^{r} .
$$

Then,

$$
0=\left\langle x ; \xi^{\alpha, \beta}-\xi^{\alpha, \gamma}\right\rangle=2\left\langle x ; e^{r}\right\rangle
$$

and therefore, $x_{r}=0$, for all $1 \leqslant r \leqslant k$. Hence, $\left\langle x ; \xi^{\alpha, \beta}\right\rangle=0$ reduces to

$$
\sum_{j=k+1}^{n} \widetilde{a}_{j}^{\alpha} x_{j}=0
$$

for all $\alpha=1, \ldots, m$. It remains to show that $x_{r}=0$, for all $k+1 \leqslant r \leqslant n$. Since $\widetilde{a}^{\alpha}=R^{T} a^{\alpha}$ for all $\alpha=1, \ldots, m$, we have

$$
\left\langle\widetilde{a}^{\alpha} ; e^{j}\right\rangle=\left\langle R^{T} a^{\alpha} ; e^{j}\right\rangle=\left\langle a^{\alpha} ; R e^{j}\right\rangle=\left\langle a^{\alpha} ; b^{j}\right\rangle=0
$$


for all $\alpha=1, \ldots, m$ and $j=1, \ldots, k$. As $R \in O(n)$,

$$
\operatorname{dim} \operatorname{span}\left\{\widetilde{a}^{\alpha} \mid \alpha=1, \ldots, m\right\}=n-k,
$$

which forces the matrix $A \in \mathbb{R}^{m \times(n-k)}$, defined by

$$
A_{\alpha \beta}=\widetilde{a}_{\beta+k}^{\alpha}, \alpha=1, \ldots, m \text { and } \beta=1, \ldots, n-k
$$

to have rank $n-k$. Now (3.3) can be written as

$$
A \widetilde{x}=0
$$

where $\widetilde{x}=\left(x_{k+1}, \ldots, x_{n}\right)$. Since the rank of $A$ is $n-k$, we get $\widetilde{x}=0$. Therefore $x=0$.

Proof of iii'): This is a consequence of the fact that

$$
\sum_{\alpha=1}^{m} t^{\alpha} a^{\alpha}=0
$$

which in turn follows from $i i$ ).

Step 3. We define $\omega \in W_{0}^{1, \infty}\left(\Omega ; \Lambda^{k}\left(\mathbb{R}^{n}\right)\right)$ by

$$
\omega(x)=u\left(R^{T} x\right) b .
$$

We now calculate $d \omega$,

$$
\begin{aligned}
d \omega(x) & =R \operatorname{grad} u\left(R^{T} x\right) \wedge b=\left(R \sum_{i=1}^{n} \frac{\partial u}{\partial x_{i}} e^{i}\right) \wedge b \\
& =\left(\sum_{i=1}^{n} \frac{\partial u}{\partial x_{i}} R e^{i}\right) \wedge b=\sum_{i=1}^{n} \frac{\partial u}{\partial x_{i}}\left(b^{i} \wedge b\right) \\
& =\sum_{i=k+1}^{n} \frac{\partial u}{\partial x_{i}}\left(b^{i} \wedge b\right) \in\left\{\sum_{i=k+1}^{n} \widetilde{a}_{i}^{\alpha}\left(b^{i} \wedge b\right)\right\}_{\alpha=1}^{m} .
\end{aligned}
$$

Using the definition of $\widetilde{a}^{\alpha}$ for all $1 \leq \alpha \leq m$, we obtain

$$
\begin{aligned}
\sum_{i=k+1}^{n} \widetilde{a}_{i}^{\alpha}\left(b^{i} \wedge b\right) & =\sum_{i=1}^{n} \widetilde{a}_{i}^{\alpha}\left(b^{i} \wedge b\right)=\left(\sum_{i=1}^{n} \widetilde{a}_{i}^{\alpha} R e^{i}\right) \wedge b \\
& =\left(R \sum_{i=1}^{n} \widetilde{a}_{i}^{\alpha} e^{i}\right) \wedge b=R \widetilde{a}^{\alpha} \wedge b=a^{\alpha} \wedge b=z^{\alpha} .
\end{aligned}
$$


Therefore, we get

$$
d \omega \in\left\{z^{\alpha} \mid \alpha=1, \ldots, m\right\} \subset E, \text { a.e. in } \Omega .
$$

Case 2: General $b^{1}, \ldots, b^{k}$.

Applying the standard Gram-Schmidt orthonormalization process to the vectors $\left\{b^{i} /\left\|b^{i}\right\| \mid i=1, \ldots, k\right\}$, we find $c^{1}, \ldots, c^{k}$ in $\mathbb{R}^{n}$ such that $c^{1}, \ldots, c^{k}$ are orthonormal and there exists $\alpha \in \mathbb{R} \backslash\{0\}$ such that

$$
\stackrel{\wedge}{\wedge} b^{i}=\alpha \stackrel{k}{\wedge} c_{i=1}^{i}
$$

More explicitly, we set $c^{1}=b^{1} /\left\|b^{1}\right\|$ and

$$
c^{r+1}=\frac{b^{r+1}-\sum_{j=1}^{r}\left\langle b^{r+1} ; c^{j}\right\rangle c^{j}}{\left\|b^{r+1}-\sum_{j=1}^{r}\left\langle b^{r+1} ; c^{j}\right\rangle c^{j}\right\|},
$$

for all $1 \leqslant r \leqslant k-1$. Then, clearly $V_{b}=V_{c}$ where

$$
c=\wedge_{i=1}^{k} c^{i} .
$$

Hence, from Case 1 , there exists $\omega \in W_{0}^{1, \infty}\left(\Omega ; \Lambda^{k}\left(\mathbb{R}^{n}\right)\right)$ such that

$$
d \omega \in E \text {, a.e. in } \Omega
$$

and $\omega$ is of the form

$$
\omega(x)=v(x) c
$$

for some $v \in W_{0}^{1, \infty}(\Omega)$. Therefore

$$
\omega(x)=u(x) b
$$

where $u \in W_{0}^{1, \infty}(\Omega)$ is defined by $u(x)=\frac{v(x)}{\alpha}$ for a.e. $x \in \Omega$. This completes the proof.

We now turn our attention to the study of necessary conditions for the existence of solutions to the differential inclusion problem. The necessary condition for $k=0$, i.e. for the gradient case, is essentially known (see [5], [6] and [12]) but it is new in all other cases, including $k=1$. The proof follows closely the method outlined in [5] and [12], so we will not present it here and we refer to [1], [2] for more details.

Theorem 3.6 [Necessary condition] Let $\Omega \subset \mathbb{R}^{n}$ be open and bounded. For $0 \leqslant k \leqslant n-1$, let $E \subseteq \Lambda^{k+1}\left(\mathbb{R}^{n}\right)$ be finite and let $\omega \in W_{0}^{1, \infty}\left(\Omega ; \Lambda^{k}\left(\mathbb{R}^{n}\right)\right)$ be such that

$$
d \omega(x) \in E \text { a.e. } x \in \Omega .
$$

Then there exists $F \subseteq E$ such that $0 \in F \cup \operatorname{ri}(\operatorname{co} F)$. 


\section{The case of the curl}

When $k=1$, i.e. for the case of the curl, we can improve the necessary condition stated in the previous section by showing that no nontrivial solutions exist in dimension less than $n-1$. Also, in this case, the sufficient condition in section 3 is, in fact, necessary. In the first subsection we prove the minimality of dimension $n-1$ and in the second one we prove the necessity and sufficiency.

Unless otherwise stated, we will assume throughout this section that $n \geqslant 3$, that $V$ is an $n$ dimensional inner product space over $\mathbb{R}$ and that $W$ is a subspace of $\Lambda^{2}(V)$.

\subsection{Minimality of dimension}

In this subsection we prove that there does not exist any nontrivial solution of the differential inclusion problem if $\operatorname{dim} \operatorname{span} E \leqslant n-2$. We start with a series of lemmas.

Lemma 4.1 Let $W$ be a non-trivial subspace of $\Lambda^{2}(V)$ and let

$$
S=\{x \in V \mid w \wedge x \neq 0 \text { for all } w \in W \backslash\{0\}\}
$$

be non-empty. Then $\mathcal{H}^{n}(V \backslash S)=0$.

Proof. Note that $V \backslash S \neq \emptyset$, because $0 \in V \backslash S$, so let $x \in V \backslash S$. Let $k$ be the dimension of $W$ and choose a basis $\left\{w_{j} \mid j=1, \ldots, k\right\}$ of $W$. Since $x \in V \backslash S$, there exists $w \in W \backslash\{0\}$ such that $w \wedge x=0$.

As $w \in W \backslash\{0\}$, there exist $\alpha_{1}, \ldots, \alpha_{k} \in \mathbb{R}$, not all zero, such that

$$
w=\sum_{j=1}^{k} \alpha_{j} w_{j}
$$

which implies that

$$
0=w \wedge x=\sum_{j=1}^{k} \alpha_{j}\left(w_{j} \wedge x\right)
$$

that is, $\left\{w_{j} \wedge x \mid j=1, \ldots, k\right\}$ is linearly dependent. Let us define a matrix $A \in \mathbb{R}^{k \times\left(\begin{array}{c}n \\ 3\end{array}\right)}$ by

$$
A_{i *}=w_{i} \wedge x
$$

where $A_{i *}$ denotes the $i$-th row of $A$, for all $i=1, \ldots, k$. Clearly $\operatorname{rank}(A) \leqslant$ $k-1$. Therefore, there exists a family $\mathcal{F}$ of polynomials generated by $k \times k$ minors of $A$ such that $M(x)=0$ for all $M \in \mathcal{F}$.

Similarly, we can also prove the converse, i.e. $M(x)=0$ for all $M \in \mathcal{F}$ implies that $x \in V \backslash S$. 
Thus, we have $x \in V \backslash S$ if and only if $M(x)=0$ for all $M \in \mathcal{F}$. Since $S \neq \emptyset$ there exists $M \in \mathcal{F}$ such that $M$ is not a zero polynomial. Therefore,

$$
\mathcal{H}^{n}(V \backslash S)=0 .
$$

To make this reasoning clearer consider the following example in $\mathbb{R}^{3}$. Assume that $\operatorname{dim} W=1$ so $W=\operatorname{span}\{w\}$ for some $w \in W \backslash\{0\}$. Given $x \in \mathbb{R}^{3} \backslash S$ we write $w=w_{12} e_{1} \wedge e_{2}+w_{13} e_{1} \wedge e_{3}+w_{23} e_{2} \wedge e_{3}$ and $x=x_{1} e_{1}+x_{2} e_{2}+x_{3} e_{3}$ with respect to the canonical basis $\left\{e_{1}, e_{2}, e_{3}\right\}$ of $\mathbb{R}^{3}$. This leads to

$$
0=w \wedge x=\left(w_{12} x_{3}+w_{23} x_{1}-w_{13} x_{2}\right) e_{1} \wedge e_{2} \wedge e_{3},
$$

hence $w_{12} x_{3}+w_{23} x_{1}-w_{13} x_{2}=0$. As $w \neq 0$, one of $w_{12}, w_{13}, w_{23}$ is non-zero. Therefore, $\mathcal{H}^{3}\left\{x \in \mathbb{R}^{3} \mid w_{12} x_{3}+w_{23} x_{1}-w_{13} x_{2}=0\right\}=0$ and so $\mathcal{H}^{3}\left(\mathbb{R}^{3} \backslash S\right)=0$. We can proceed similarly when $\operatorname{dim} W=2$.

Lemma 4.2 Assume that $x \neq 0,1 \leqslant \operatorname{dim} W(x) \leqslant n-2$ and let

$$
S=\left\{u \in V / \mathbb{R} x \mid w^{\star} \wedge u \neq 0 \text { for all } w^{\star} \in W_{x}^{\star} \backslash\{0\}\right\} .
$$

If $\mathcal{H}^{n-1}((V / \mathbb{R} x) \backslash S)=0$, then there exists $z \in V \backslash\{0\}$ such that $w \wedge z \neq 0$ for all $w \in W \backslash\{0\}$.

Proof. Let $r=\operatorname{dim} W(x), 1 \leqslant r \leqslant n-2$, and let $\left\{x \wedge y_{j} \mid j=1, \ldots, r\right\}$ be a basis of $W(x)$. Then $B=\left\{x, y_{1}, \ldots, y_{r}\right\}$ is linearly independent so we can extend $B$ to a basis $\left\{x, y_{1}, \ldots, y_{n-1}\right\}$ of $V$ such that

i) $y_{n-1} \perp\left\{x, y_{1}, \ldots, y_{n-2}\right\}$

ii) $\left\|y_{n-1}\right\|=1$.

Since $\mathcal{H}^{n-1}((V / \mathbb{R} x) \backslash S)=0$ and $\operatorname{dim}(V / \mathbb{R} x)=n-1$, it is possible to choose $u \in$ $S$ such that $u \notin \operatorname{span}\left\{\pi_{x}\left(y_{1}\right), \ldots, \pi_{x}\left(y_{n-2}\right)\right\}$. For this $u$ we find $z=\sum_{j=1}^{n-1} \lambda_{j} y_{j} \in$ $\operatorname{span}\left\{y_{1}, \ldots, y_{n-1}\right\}$ such that $\pi_{x}(z)=u$. We claim that $\left\langle z ; y_{n-1}\right\rangle \neq 0$. Indeed, if $\left\langle z ; y_{n-1}\right\rangle=0$, we would have $\left\langle z ; y_{n-1}\right\rangle=\lambda_{n-1}=0$, because of the way $y_{n-1}$ was chosen. Hence $z=\sum_{j=1}^{n-2} \lambda_{j} y_{j}$ and so $u \in \operatorname{span}\left\{\pi_{x}\left(y_{1}\right), \ldots, \pi_{x}\left(y_{n-2}\right)\right\}$, contradicting the choice of $u$.

We will now prove that $w \wedge z \neq 0$ for all $w \in W \backslash\{0\}$ by showing that if $w \wedge z=0$, for some $w \in W$, then $w=0$.

If $w \wedge z=0$ there exists $b \in V$ such that $w=b \wedge z$, which implies that $\pi_{x}^{\star}(w) \wedge u=0$. Since $u \in S$ and $\pi_{x}^{\star}(w) \in W_{x}^{\star}$, it follows that $\pi_{x}^{\star}(w)=0$, which in turn implies that $w \in W(x)$. Thus, there exist $\beta_{1}, \ldots, \beta_{r} \in \mathbb{R}$ such that

$$
w=\sum_{i=1}^{r} \beta_{i}\left(x \wedge y_{i}\right) .
$$


As $z=\sum_{j=1}^{n-1} \lambda_{j} y_{j}$, for some $\lambda_{1}, \ldots, \lambda_{n-1} \in \mathbb{R}$, we have

$$
\left(\sum_{i=1}^{r} \beta_{i}\left(x \wedge y_{i}\right)\right) \wedge\left(\sum_{j=1}^{n-1} \lambda_{j} y_{j}\right)=0 .
$$

From the linear independence of $\left\{x \wedge y_{i} \wedge y_{j} \mid i<j\right\}$, the coefficients of $x \wedge y_{i} \wedge$ $y_{n-1}$ are equal to zero, for all $i=1, \ldots, r$, so

$$
\beta_{i} \lambda_{n-1}=0 \text { for all } i=1, \ldots, r .
$$

However, we showed that $\lambda_{n-1} \neq 0$, so $\beta_{i}=0$ for all $i=1, \ldots, r$ which yields $w=0$.

Lemma 4.3 Let $W$ be a subspace of $\Lambda^{2}(V)$ with $1 \leqslant \operatorname{dim} W \leqslant n-2$ and let

$$
S_{n}=\{x \in V \mid w \wedge x \neq 0 \text { for all } w \in W \backslash\{0\}\} .
$$

Then $\mathcal{H}^{n}\left(V \backslash S_{n}\right)=0$.

Proof. The proof is by induction on $n$.

Step 1. Induction hypothesis $(n=3)$ :

In this case, $W$ has dimension 1 . Let $W=\operatorname{span}\left\{w_{1}\right\}$, for some $w_{1} \in W \backslash\{0\}$, and $x \in V \backslash\{0\}$.

If $w_{1} \wedge x \neq 0$, then clearly $w \wedge x \neq 0$ for all $w \in W \backslash\{0\}$ and hence $x \in S_{3}$. Suppose now that $w_{1} \wedge x=0$. Then there exists $v \in V \backslash\{0\}$ such that $w_{1}=x \wedge v$. We choose $z \perp\{x, v\}, z \neq 0$. It is easy to see that $w \wedge z \neq 0$, for all $w \in W \backslash\{0\}$, and thus $z \in S_{3}$.

Therefore $S_{3} \neq \emptyset$ and so, by virtue of Lemma $4.1, \mathcal{H}^{3}\left(V \backslash S_{3}\right)=0$.

\section{Step 2. Induction step:}

Suppose the lemma is true for $n=k-1$, we prove it also holds for $n=k$.

Let $V^{k}$ be a $k$-dimensional inner product space over $\mathbb{R}$, let $W^{k}$ be a subspace of $\Lambda^{2}\left(V^{k}\right)$ with $1 \leqslant \operatorname{dim} W^{k} \leqslant k-2$ and let

$$
S_{k}=\left\{x \in V^{k} \mid w \wedge x \neq 0 \text { for all } w \in W^{k} \backslash\{0\}\right\} .
$$

Let $x \in V^{k}$ be such that $\|x\|=1$ and $W^{k}(x) \neq W^{k}$, such an $x$ exists because $\operatorname{dim} W^{k} \leqslant k-2$. Recall that

$$
W^{k}(x)=\left\{w \in W^{k} \mid w \wedge x=0\right\} \text { and } W_{x}^{k \star}=\pi_{x}^{\star}\left(W^{k}\right) .
$$


If $W^{k}(x)=\{0\}$, then $w \wedge x \neq 0$ for all $w \in W^{k} \backslash\{0\}$ and so $x \in S_{k}$. By Lemma 4.1, we conclude that $\mathcal{H}^{k}\left(V^{k} \backslash S_{k}\right)=0$.

If $W^{k}(x) \neq\{0\}$, as $W^{k}(x) \varsubsetneqq W^{k}$, we have $1 \leqslant \operatorname{dim} W^{k}(x)<\operatorname{dim} W^{k} \leqslant$ $k-2$ from which we obtain

$$
1 \leqslant \operatorname{dim} W_{x}^{k \star}=\operatorname{dim} W^{k}-\operatorname{dim} W^{k}(x) \leqslant k-3 .
$$

By the induction hypothesis, it follows that

$$
\mathcal{H}^{k-1}\left(\left(V^{k} / \mathbb{R} x\right) \backslash S_{k-1}\right)=0
$$

where $S_{k-1}=\left\{u \in V^{k} / \mathbb{R} x \mid w^{\star} \wedge u \neq 0\right.$, for all $\left.w^{\star} \in W_{x}^{k \star} \backslash\{0\}\right\}$. Therefore Lemma 4.2 guarantees the existence of $z \in V^{k} \backslash\{0\}$ such that $w \wedge z \neq 0$, for all $w \in W^{k} \backslash\{0\}$. This shows that $S_{k} \neq \emptyset$ so, employing once again Lemma 4.1, we get

$$
\mathcal{H}^{k}\left(V^{k} \backslash S_{k}\right)=0
$$

This completes the induction step and finishes the proof of the lemma.

Corollary 4.4 Let $W$ be a subspace of $\Lambda^{2}\left(\mathbb{R}^{n}\right)$ such that $W \cap\left(x \wedge \mathbb{R}^{n}\right) \neq\{0\}$ for all $x \in \mathcal{S} \subset \mathbb{R}^{n}$ with $\mathcal{H}^{n}(\mathcal{S})>0$. Then $\operatorname{dim} W \geqslant n-1$.

Proof. Suppose, on the contrary, that $\operatorname{dim} W \leqslant n-2$ and let

$$
\mathcal{T}=\left\{x \in \mathbb{R}^{n} \mid w \wedge x \neq 0 \text { for all } w \in W \backslash\{0\}\right\} .
$$

From lemma 4.3, $\mathcal{H}^{n}\left(\mathbb{R}^{n} \backslash \mathcal{T}\right)=0$. Since $\mathcal{H}^{n}(\mathcal{S})>0$, we have $\mathcal{T} \cap \mathcal{S} \neq \emptyset$. Choose $x_{0} \in \mathcal{T} \cap \mathcal{S}$. Since $x_{0} \in \mathcal{S}$, there exists $w \in W \backslash\{0\}$ such that $w \wedge x_{0}=0$, which contradicts the fact that $x_{0} \in \mathcal{T}$. Therefore, $\operatorname{dim} W \geqslant n-1$.

We now come to the main theorem of this subsection.

Theorem 4.5 Let $n \geqslant 3, \Omega$ be a bounded, open set in $\mathbb{R}^{n}, E$ be a subset of $\Lambda^{2}\left(\mathbb{R}^{n}\right)$ such that $0 \notin E$ and $u \in W_{0}^{1, \infty}\left(\Omega ; \mathbb{R}^{n}\right)$ be a solution of the problem

$$
\operatorname{curl} u \in E \text { a.e. in } \Omega \text {. }
$$

Then $\operatorname{dim} \operatorname{span} E \geqslant n-1$.

Proof. Let $\mathbb{P}: \Lambda^{2}\left(\mathbb{R}^{n}\right) \rightarrow \Lambda^{2}\left(\mathbb{R}^{n}\right)$ denote the projection onto the orthogonal complement of span $E$.

Since $u \in W_{0}^{1, \infty}\left(\Omega ; \mathbb{R}^{n}\right)$, extending $u$ by 0 to $\mathbb{R}^{n}$, we have

$$
\mathbb{P}(\operatorname{curl} u(x))=0 \text { for a.e. } x \in \mathbb{R}^{n} .
$$

Applying the Fourier transform, we obtain

$$
\mathbb{P}(x \wedge \hat{u}(x))=0 \text { for a.e. } x \in \mathbb{R}^{n},
$$


which implies that

$$
x \wedge \hat{u}(x) \in \operatorname{span} E \text { for a.e. } x \in \mathbb{R}^{n} .
$$

Since $0 \notin E$, there exists a subset $\mathcal{S} \subset \mathbb{R}^{n}$ such that $\mathcal{H}^{n}(\mathcal{S})>0$ and $x \wedge \hat{u}(x) \neq 0$ for all $x \in \mathcal{S}$. Indeed, if not, we would have $x \wedge \hat{u}(x)=0$ for a.e. $x \in \mathbb{R}^{n}$ which implies, from Plancherel Theorem, that $\operatorname{curl} u=0$ a.e. in $\Omega$, contradicting the assumption that $0 \notin E$. Therefore, from Corollary 4.4,

$$
\operatorname{dim} \operatorname{span}\{x \wedge \hat{u}(x) \mid x \in \mathcal{S}\} \geqslant n-1
$$

and so $\operatorname{dim} \operatorname{span} E \geqslant n-1$.

\subsection{Necessity and sufficiency in dimension $n-1$}

In this subsection, we begin by characterizing all $n-1$ dimensional subspaces $W$ of $\Lambda^{2}(V)$ with the property that $W \cap(x \wedge V) \neq\{0\}$ for all $x \in \mathcal{S}$ for some $\mathcal{S} \subseteq V$ with $\mathcal{H}^{n}(\mathcal{S})>0$.

Recall that for $b \in V$ we define

$$
V_{b}=\{x \wedge b \mid x \in V\} \subseteq \Lambda^{2}(V) .
$$

Our goal is to prove the following theorem.

Theorem 4.6 [Algebraic version] Let $W$ be an $n-1$ dimensional subspace of $\Lambda^{2}(V)$. Then $W \cap(x \wedge V) \neq\{0\}$ for all $x \in \mathcal{S}$, for some $\mathcal{S} \subseteq V$ with $\mathcal{H}^{n}(\mathcal{S})>0$, if and only if there exists $b \in V \backslash\{0\}$ such that $W=V_{b}$.

We start with the following lemma which states that $\mathcal{S}$ can be replaced by $V \backslash\{0\}$.

Lemma 4.7 Let $W$ be such that $\operatorname{dim} W=n-1$ and $W \cap(x \wedge V) \neq\{0\}$ for all $x \in \mathcal{S}$, for some $\mathcal{S} \subseteq V$ with $\mathcal{H}^{n}(\mathcal{S})>0$. Then, $W \cap(x \wedge V) \neq\{0\}$ for all $x \in V \backslash\{0\}$.

Proof. Suppose there exists $y \in V \backslash\{0\}$ such that $w \wedge y \neq 0$ for all $w \in W \backslash\{0\}$. Let $\mathbb{B}=\{x \in V \mid w \wedge x \neq 0$ for all $w \in W \backslash\{0\}\}$. Then $\mathbb{B} \neq \emptyset$ so, using Lemma 4.1, we have $\mathcal{H}^{n}(V \backslash \mathbb{B})=0$. But this is a contradiction because $\mathcal{S} \subseteq V \backslash \mathbb{B}$ and $\mathcal{H}^{n}(\mathcal{S})>0$. Hence, $\mathbb{B}=\emptyset$.

With Lemma 4.7 in hand, we have an equivalent version of Theorem 4.6.

Theorem 4.8 [Algebraic Version] Let $W$ be an $n-1$ dimensional subspace of $\Lambda^{2}(V)$. Then $W \cap(x \wedge V) \neq\{0\}$ for all $x \in V \backslash\{0\}$ if and only if there exists $b \in V \backslash\{0\}$ such that $W=V_{b}$. 
In order to prove Theorem 4.8 we begin with a weaker version.

Lemma 4.9 [Weak Version] Let $W$ be an $n-1$ dimensional subspace of $\Lambda^{2}(V)$ such that $W \cap(x \wedge V) \neq\{0\}$ for all $x \in V \backslash\{0\}$. Then exactly one of the following two possibilities can occur:

i) there exists $b \in V \backslash\{0\}$ such that $W=V_{b}$

ii) $\operatorname{dim}\{w \in W \mid w \wedge b=0\}=1$ for all $b \in V \backslash\{0\}$.

Proof. If $n=3$ it is easy to see that any 2 dimensional subspace $W$ of $\Lambda^{2}(V)$ is of the form $V_{b}$, for some $b \in V \backslash\{0\}$. Hence, we assume that $n \geqslant 4$. Suppose neither $i$ ) nor $i i$ ) hold. Then, there exists $x \in V \backslash\{0\}$ such that $1<\operatorname{dim} W(x)<n-1$ where

$$
W(x)=\{w \in W \mid w \wedge x=0\} .
$$

Recall that $\pi_{x}^{\star}: \Lambda^{2}(V) \rightarrow \Lambda^{2}(V / \mathbb{R} x)$ is the epimorphism induced by the canonical epimorphism $\pi_{x}: V \rightarrow V / \mathbb{R} x$ and that $W_{x}^{\star}=\pi_{x}^{\star}(W)$.

Since $n-2 \geqslant \operatorname{dim} W(x) \geqslant 2$, we have $1 \leqslant \operatorname{dim} W_{x}^{\star} \leqslant n-3$. Hence from Lemma $4.3, \mathcal{H}^{n-1}\left((V / \mathbb{R} x) \backslash S_{n-1}\right)=0$, where

$$
S_{n-1}=\left\{u \in V / \mathbb{R} x \mid w^{\star} \wedge u \neq 0 \text { for all } w^{\star} \in W_{x}^{\star} \backslash\{0\}\right\} .
$$

By Lemma 4.2, there exists $z \in V \backslash\{0\}$ such that $w \wedge z \neq 0$, for all $w \in W \backslash\{0\}$. But this leads to the conclusion that $W \cap(z \wedge V)=\{0\}$, which contradicts the hypothesis that $W \cap(z \wedge V) \neq\{0\}$. This completes the proof of the lemma.

We will now show that the second possibility in Lemma 4.9 can, in fact, never hold, which will finish the proof of Theorem 4.8. In other words, we prove by induction, that

Lemma 4.10 There does not exist any $n-1$ dimensional subspace $W$ of $\Lambda^{2}(V)$ such that $\operatorname{dim}\{w \in W \mid w \wedge b=0\}=1$ for all $b \in V \backslash\{0\}$.

Before we embark on the proof of Lemma 4.10 we need two technical lemmas, the first of which, in essence, helps us to make a careful choice in the proof of Lemma 4.10, whereas the second one provides the induction step.

Lemma 4.11 Let $n \geqslant 4$. There does not exist any $n-1$ dimensional subspace $W$ of $\Lambda^{2}(V)$ with the properties that

i) for each $b \in V \backslash\{0\}$, there exists $x_{b} \in V$ such that $b \wedge x_{b} \neq 0$ and $W \wedge b \wedge x_{b}=\{0\}$ and

ii) $\operatorname{dim}\{w \in W \mid w \wedge b=0\}=1$ for all $b \in V \backslash\{0\}$. 
Proof. We will arrive at a contradiction by assuming such a $W$ exists. Let $c \in V \backslash\{0\}$ and let $x_{c} \in V \backslash\{0\}$ be such that $c \wedge x_{c} \neq 0$ and $W \wedge c \wedge x_{c}=\{0\}$. Without loss of generality we can assume that $c \perp x_{c}$. Let $b \perp\left\{c, x_{c}\right\}, b \neq 0$, and let $x_{b} \in V \backslash\{0\}$ be such that $W \wedge b \wedge x_{b}=\{0\}$ and $b \perp x_{b}$. Now two cases can arise:

1. $\operatorname{span}\left\{b, x_{b}\right\} \cap \operatorname{span}\left\{c, x_{c}\right\}=\{0\}$ or

2. $\operatorname{dim}\left(\operatorname{span}\left\{b, x_{b}\right\} \cap \operatorname{span}\left\{c, x_{c}\right\}\right)=1$.

Case 1. $\operatorname{span}\left\{b, x_{b}\right\} \cap \operatorname{span}\left\{c, x_{c}\right\}=\{0\}$

Clearly $\left\{b, c, x_{b}, x_{c}\right\}$ are linearly independent. Let $\nu_{b+c} \in V \backslash\{0\}$ be a fixed vector such that $(b+c) \wedge \nu_{b+c} \in W \backslash\{0\}$. Then

$$
(b+c) \wedge \nu_{b+c} \wedge b \wedge x_{b}=0
$$

and

$$
c \wedge \nu_{b+c} \wedge b \wedge x_{b}=0 .
$$

Since $\left\{c, b, x_{b}\right\}$ are linearly independent, it follows from (4.2) that there exist $\alpha_{1}, \beta_{1}, \gamma_{1} \in \mathbb{R}$, not all zero, such that $\nu_{b+c}=\alpha_{1} c+\beta_{1} b+\gamma_{1} x_{b}$.

Similarly,

$$
(b+c) \wedge \nu_{b+c} \wedge c \wedge x_{c}=0
$$

and

$$
b \wedge \nu_{b+c} \wedge c \wedge x_{c}=0
$$

from which we conclude that $\nu_{b+c}=\alpha_{2} b+\beta_{2} c+\gamma_{2} x_{c}$ for some $\alpha_{2}, \beta_{2}, \gamma_{2} \in \mathbb{R}$, not all zero. Therefore,

$$
\left(\beta_{1}-\alpha_{2}\right) b+\left(\alpha_{1}-\beta_{2}\right) c+\gamma_{1} x_{b}-\gamma_{2} x_{c}=0 .
$$

By the linear independence of $\left\{b, c, x_{b}, x_{c}\right\}$ we obtain $\beta_{1}=\alpha_{2}, \alpha_{1}=\beta_{2}, \gamma_{1}=$ $\gamma_{2}=0$. Thus $\nu_{b+c}=\alpha b+\beta c$ for some $\alpha, \beta \in \mathbb{R}$, not both zero, and so,

$$
(b+c) \wedge \nu_{b+c}=(b+c) \wedge(\alpha b+\beta c)=(\beta-\alpha) b \wedge c \in W \backslash\{0\} .
$$

In other words

$$
b \wedge c \in W \backslash\{0\} .
$$

Now let $\nu_{b+x_{c}} \in V \backslash\{0\}$ be a fixed vector such that $\left(b+x_{c}\right) \wedge \nu_{b+x_{c}} \in$ $W \backslash\{0\}$. Proceeding exactly as before, since $\left(b+x_{c}\right) \wedge \nu_{b+x_{c}} \wedge b \wedge x_{b}=0$ and $\left(b+x_{c}\right) \wedge \nu_{b+x_{c}} \wedge c \wedge x_{c}=0$, we find that

$$
b \wedge x_{c} \in W \backslash\{0\} .
$$


But, as $\left\{b, c, x_{c}\right\}$ are linearly independent, $\left\{b \wedge c, b \wedge x_{c}\right\}$ are also linearly independent. Thus, using equations (4.3) and (4.4), we see that

$$
\operatorname{dim}\{w \in W \mid w \wedge b=0\} \geqslant 2
$$

which contradicts the hypothesis of the lemma.

Case 2: $\operatorname{dim}\left(\operatorname{span}\left\{b, x_{b}\right\} \cap \operatorname{span}\left\{c, x_{c}\right\}\right)=1$

Let $\operatorname{span}\left\{b, x_{b}\right\} \cap \operatorname{span}\left\{c, x_{c}\right\}=\operatorname{span}\{t\}$ for some $t \in V \backslash\{0\}$. Since $t \in$ $\operatorname{span}\left\{b, x_{b}\right\}$ there exist $\alpha_{1}, \beta_{1} \in \mathbb{R}$ such that $t=\alpha_{1} b+\beta_{1} x_{b}$. Similarly, there exist $\alpha_{2}, \beta_{2} \in \mathbb{R}$ such that $t=\alpha_{2} c+\beta_{2} x_{c}$. Therefore

$$
\alpha_{1} b+\beta_{1} x_{b}=\alpha_{2} c+\beta_{2} x_{c} .
$$

Taking the inner product with $b$ on both sides of the above equation, and noting that $b \perp\left\{x_{b}, c, x_{c}\right\}$, we get $\alpha_{1}=0$. Thus $t=\beta_{1} x_{b}$, where $\beta_{1} \neq 0$ since $t \neq 0$, and so $x_{b} \in \operatorname{span}\left\{c, x_{c}\right\}$. Choose a vector $t_{0} \in V \backslash\{0\}$ such that $t_{0} \perp\left\{b, c, x_{c}\right\}$, notice that $t_{0} \perp x_{b}$.

Since $t_{0}, x_{b} \neq 0$ and $t_{0} \perp x_{b}$ evidently $t_{0} \wedge x_{b} \neq 0$. Let $\nu_{0} \in V \backslash\{0\}$ be a fixed vector such that $t_{0} \wedge \nu_{0} \in W \backslash\{0\}$. We can assume that $\nu_{0} \perp t_{0}$. As in case 1, since $t_{0} \wedge \nu_{0} \wedge b \wedge x_{b}=0$ and $t_{0} \wedge \nu_{0} \wedge c \wedge x_{c}=0$, we deduce that

$$
\nu_{0} \in \operatorname{span}\left\{b, x_{b}\right\} \cap \operatorname{span}\left\{c, x_{c}\right\}=\operatorname{span}\{t\}=\operatorname{span}\left\{x_{b}\right\}
$$

which implies the existence of $\rho \in \mathbb{R} \backslash\{0\}$ such that $\nu_{0}=\rho x_{b}$. As $t_{0} \wedge \nu_{0} \in$ $W \backslash\{0\}$, it follows that

$$
t_{0} \wedge x_{b} \in W \backslash\{0\} .
$$

Repeating the above argument, we can also show that

$$
\left(t_{0}+b\right) \wedge x_{b} \in W \backslash\{0\} .
$$

Equations (4.5) and (4.6) imply that $b \wedge x_{b} \in W \backslash\{0\}$ so we conclude that the linearly independent vectors $b \wedge x_{b}$ and $t_{0} \wedge x_{b}$ both belong to $W \backslash\{0\}$. However, this yields

$$
\operatorname{dim}\left\{w \in W \mid w \wedge x_{b}=0\right\} \geqslant 2,
$$

contradicting the hypothesis that $\operatorname{dim}\{w \in W \mid w \wedge b=0\}=1$ for all $b \in$ $V \backslash\{0\}$.

Lemma 4.12 Let $n \geqslant 4$ and let $W$ be an $n-1$ dimensional subspace of $\Lambda^{2}(V)$ such that

$$
\operatorname{dim}\{w \in W \mid w \wedge b=0\}=1 \text { for all } b \in V \backslash\{0\} .
$$

Let $c \in V \backslash\{0\}$ and set $W_{c}^{\star}=\pi_{c}^{\star}(W)$. Then, $W_{c}^{\star} \cap(u \wedge(V / \mathbb{R} c)) \neq\{0\}$ for all $u \in(V / \mathbb{R} c) \backslash\{0\}$. 
Proof. Suppose the result does not hold, that is,

$$
S_{n-1}=\left\{u \in V / \mathbb{R} c \mid w^{\star} \wedge u \neq 0 \text { for all } w^{\star} \in W_{c}^{\star} \backslash\{0\}\right\} \neq \emptyset .
$$

By Lemma 4.1, we conclude that $\mathcal{H}^{n-1}\left((V / \mathbb{R} c) \backslash S_{n-1}\right)=0$. Let

$$
W(c)=\{w \in W \mid w \wedge c=0\} .
$$

By hypothesis $\operatorname{dim} W(c)=1$ so we can apply Lemma 4.2 to obtain $z \in V \backslash\{0\}$ such that $w \wedge z \neq 0$, for all $w \in W \backslash\{0\}$. But this contradicts the fact that $\operatorname{dim}\{w \in W \mid w \wedge z=0\}=1$ and the lemma is proved.

We are now in position to prove Lemma 4.10.

Proof of lemma 4.10. We prove by induction.

For $n=3$, the lemma is evidently true because any 2-dimensional subspace $W$ of $\Lambda^{2}(V)$ is of the form $V_{b}$, for some $b \in V \backslash\{0\}$.

Let us assume that the lemma is true for $n=k$, we prove it for $n=k+1$. We argue by contradiction and assume there exists a $k$ dimensional subspace $W$ of $\Lambda^{2}(V)$, where $\operatorname{dim} V=k+1$, such that

$$
\operatorname{dim} W(b)=\operatorname{dim}\{w \in W \mid w \wedge b=0\}=1 \text { for all } b \in V \backslash\{0\} .
$$

We choose, by virtue of Lemma $4.11, b \in V \backslash\{0\}$ such that $W \wedge b \wedge c \neq\{0\}$ for all $c \in V$ such that $b \wedge c \neq 0$. Let $\pi_{b}^{\star}: \Lambda^{2}(V) \rightarrow \Lambda^{2}(V / \mathbb{R} b)$ be the epimorphism induced by the canonical epimorphism $\pi_{b}: V \rightarrow V / \mathbb{R} b$ and let $W_{b}^{\star}=\pi_{b}^{\star}(W)$. Then, $\operatorname{dim} W_{b}^{\star}=k-1$ and, using Lemma 4.12, we have $W_{b}^{\star} \cap(u \wedge(V / \mathbb{R} b)) \neq\{0\}$ for all $u \in(V / \mathbb{R} b) \backslash\{0\}$. Therefore, invoking Lemma 4.9, two possibilities may occur:

A) there exists $u \in(V / \mathbb{R} b) \backslash\{0\}$ such that $W_{b}^{\star}=(V / \mathbb{R} b)_{u}$ or

B) $\operatorname{dim}\left\{w^{\star} \in W_{b}^{\star} \mid w^{\star} \wedge p=0\right\}=1$ for all $p \in(V / \mathbb{R} b) \backslash\{0\}$.

We will show that either possibility leads to a contradiction.

Indeed, suppose there exists $u \in(V / \mathbb{R} b) \backslash\{0\}$ such that $W_{b}^{\star}=(V / \mathbb{R} b)_{u}$. We choose $z \in \operatorname{span}\{b\}^{\perp}$ such that $u=\pi_{b}(z)$. Then $b \wedge z \neq 0$ and for each $w \in W$, as $\pi_{b}^{\star}(w) \in W_{b}^{\star}=(V / \mathbb{R} b)_{u}$, there exists $p_{w} \in V$ such that

$$
\pi_{b}^{\star}(w)=\pi_{b}\left(p_{w}\right) \wedge u=\pi_{b}\left(p_{w}\right) \wedge \pi_{b}(z)=\pi_{b}^{\star}\left(p_{w} \wedge z\right) .
$$

Thus, $w-p_{w} \wedge z \in$ ker $\pi_{b}^{\star}$ and so there exists $q_{w} \in V$ so that $w=p_{w} \wedge z+q_{w} \wedge b$. Therefore, $w \wedge b \wedge z=0$ for all $w \in W$, i.e. $W \wedge b \wedge z=\{0\}$. But this contradicts the way $b$ was chosen.

On the other hand, if case B) were to hold, we would have a $k-1$ dimensional subspace $W_{b}^{\star}$ of $\Lambda^{2}(V / \mathbb{R} b), \operatorname{dim}(V / \mathbb{R} b)=k$, such that

$$
\operatorname{dim}\left\{w^{\star} \in W_{b}^{\star} \mid w^{\star} \wedge p=0\right\}=1, \text { for all } p \in(V / \mathbb{R} b) \backslash\{0\},
$$


contradicting the induction assumption that the lemma is true for $n=k$.

This completes the induction step and the proof of the lemma.

We now employ these algebraic preliminaries to prove the main theorem of this subsection.

Theorem 4.13 [Main theorem for the curl] Let $n \geqslant 3, \Omega \subset \mathbb{R}^{n}$ be open, bounded and let $E \subset \Lambda^{2}\left(\mathbb{R}^{n}\right)$ be such that $0 \notin E$ and $\operatorname{dim} \operatorname{span} E=n-1$. Then, there exists a solution $u \in W_{0}^{1 \infty}\left(\Omega ; \mathbb{R}^{n}\right)$ to the problem

$$
\operatorname{curl} u \in E \text { a.e. in } \Omega
$$

if and only if $0 \in \operatorname{ri}(\operatorname{co} E)$ and there exists $b \in \mathbb{R}^{n} \backslash\{0\}$ such that $\operatorname{span} E=$ $\left(\mathbb{R}^{n}\right)_{b}$.

Proof. If $0 \in \operatorname{ri}(\operatorname{co} E)$ and there exists $b \in \mathbb{R}^{n} \backslash\{0\}$ such that span $E=\left(\mathbb{R}^{n}\right)_{b}$, we refer to Theorem 3.2 for the construction of the solution. We will prove the converse.

Let $u \in W_{0}^{1 \infty}\left(\Omega ; \mathbb{R}^{n}\right)$ be a solution of $(4.7)$. Let $\mathbb{P}: \Lambda^{2}\left(\mathbb{R}^{n}\right) \rightarrow \Lambda^{2}\left(\mathbb{R}^{n}\right)$ denote the projection onto the orthogonal complement of $\operatorname{span} E$. Since $u \in$ $W_{0}^{1, \infty}\left(\Omega ; \mathbb{R}^{n}\right)$, extending $u$ by 0 to $\mathbb{R}^{n}$, we have

$$
\mathbb{P}(\operatorname{curl} u(x))=0 \text {, for a.e. } x \in \mathbb{R}^{n} \text {. }
$$

Applying the Fourier transform, we obtain

$$
\mathbb{P}(x \wedge \hat{u}(x))=0 \text {, for a.e. } x \in \mathbb{R}^{n},
$$

which implies that

$$
x \wedge \hat{u}(x) \in \operatorname{span} E \text {, for a.e. } x \in \mathbb{R}^{n} \text {. }
$$

Now because $0 \notin E$, there exists a subset $\mathcal{S} \subset \mathbb{R}^{n}$ such that $\mathcal{H}^{n}(\mathcal{S})>0$ and $x \wedge \hat{u}(x) \neq 0$ for all $x \in \mathcal{S}$. Therefore, from Theorem 4.6, it follows that there exists $b \in \mathbb{R}^{n} \backslash\{0\}$ such that $\operatorname{span} E=\left(\mathbb{R}^{n}\right)_{b}$.

It remains to show that $0 \in \operatorname{ri}(\operatorname{co} E)$. From Jensen's inequality we can deduce that $0 \in \overline{\mathrm{co}} E$. We now prove that 0 belongs, in fact, to $\operatorname{ri}(\operatorname{co} E)$. Suppose not, that is, suppose $0 \in \operatorname{rbd}(\operatorname{co} E)$.

Let $a_{1}, \ldots, a_{m} \in \Lambda^{2}\left(\mathbb{R}^{n}\right)$ be an orthonormal basis of $(\operatorname{span} E)^{\perp}$ where $m=$ $\left(\begin{array}{l}n \\ 2\end{array}\right)-n+1$. Since $0 \in \operatorname{rbd}(\operatorname{co} E)$, there exists $b \in \operatorname{span} E$ such that $\|b\|=1$ and

$$
\langle w ; b\rangle \geqslant 0, \text { for all } w \in \overline{\mathrm{co}} E .
$$

In particular, $\langle\operatorname{curl} u(x) ; b\rangle \geqslant 0$ for a.e. $x \in \Omega$. But, as $u \in W_{0}^{1 \infty}\left(\Omega, \mathbb{R}^{n}\right)$,

$$
\int_{\Omega}\langle\operatorname{curl} u(x) ; b\rangle=0
$$


$\mathrm{SO}$

$$
\langle\operatorname{curl} u(x) ; b\rangle=0 \text { for a.e. } x \in \Omega \text {. }
$$

Hence, $\langle\operatorname{curl} u(x) ; w\rangle=0$ for the $m+1$ linearly independent vectors $w=$ $b, a_{1}, \ldots, a_{m}$ in $\Lambda^{2}\left(\mathbb{R}^{n}\right)$ and for a.e. $x \in \Omega$. Letting

$$
\Omega_{0}=\{x \in \Omega \mid \operatorname{curl} u(x) \in E \text { and }\langle\operatorname{curl} u(x) ; b\rangle=0\}
$$

we have

$$
\operatorname{dim} \operatorname{span} \operatorname{curl} u\left(\Omega_{0}\right) \leqslant\left(\begin{array}{l}
n \\
2
\end{array}\right)-m-1=n-2 .
$$

But this, together with the fact that $0 \notin \operatorname{curl} u\left(\Omega_{0}\right)$, contradicts Theorem 4.5. Hence $0 \in \operatorname{ri}(\operatorname{co} E)$.

Remark 4.14 Note that, in contrast with Theorem 3.6, we do not require $E$ to be finite here.

\subsection{The curl in dimension three}

As before, we are interested in the problem of finding $u \in W_{0}^{1, \infty}\left(\Omega ; \mathbb{R}^{3}\right)$ satisfying

$$
\operatorname{curl} u \in E \text { a.e. in } \Omega \text {. }
$$

In the next theorem we assume that $0 \notin E$ to avoid the trivial case.

Theorem 4.15 Let $\Omega \subset \mathbb{R}^{3}$ be open, bounded and let $E \subset \mathbb{R}^{3} \backslash\{0\}$. Then (4.8) has a solution if and only if there exists a subset $F \subseteq E$, such that $\operatorname{dim} \operatorname{span} F \geqslant 2$ and $0 \in \operatorname{ri}(\operatorname{co} F)$.

Remark 4.16 (i) It is the sufficiency part which is more interesting here. When $\operatorname{dim} \operatorname{span} E=2$, Theorem 4.15 is just a restatement of Theorem 4.13 because we can take $F=E$. However, this sufficient condition is also true when $\operatorname{dim} \operatorname{span} E=3$ so this theorem improves the aforementioned one. It also extends previous results of [17], which do not consider Dirichlet boundary conditions, and of [3], [9], where a non-constructive proof was obtained only for the case $\operatorname{dim} \operatorname{span} F=2$.

(ii) The necessary part, according to the referee, is implicit in [17]. Nevertheless, our results enable us to provide a more elementary proof so we will outline it below, for the sake of both simplicity and completeness.

Proof. Sufficient Condition. If $\operatorname{dim} \operatorname{span} F=2$, as any 2-dimensional subspace of $\mathbb{R}^{3}$ is of the form $\left(\mathbb{R}^{3}\right)_{b}$ for some $b \in \mathbb{R}^{3} \backslash\{0\}$, we apply Theorem 4.13 to $F$ in order to obtain a solution. 
Thus, the theorem will be proved once we show the existence of solutions when $\operatorname{dim} \operatorname{span} F=3$ (i.e. $\operatorname{dim} \operatorname{span} F=\operatorname{dim} \operatorname{span} E$ ).

We divide this proof in two steps. We note that it is enough to prove it for a particular open set $\Omega$, the general case follows by virtue of Vitali's covering theorem.

Step 1. Applying Lemma 3.4 we find $z^{\alpha} \in F, t^{\alpha}>0, \alpha=1,2,3,4$ such that

$$
\sum_{\alpha=1}^{4} t^{\alpha} z^{\alpha}=0, \sum_{\alpha=1}^{4} t^{\alpha}=1, \operatorname{dim} \operatorname{span}\left\{z^{1}, z^{2}, z^{3}, z^{4}\right\}=3 .
$$

Without loss of generality we can assume that $\left\{z^{1}, z^{2}, z^{3}\right\}$ are linearly independent. Now, choosing $\epsilon>0$ sufficiently small so that $t_{2}>\epsilon$, we write

$$
\begin{aligned}
0=t^{1} z^{1} & +\left(t^{2}-\epsilon\right) z^{2} \\
& +\left(t^{3}+t^{4}+\epsilon\right)\left[\frac{\epsilon}{t^{3}+t^{4}+\epsilon} z^{2}+\frac{t^{3}}{t^{3}+t^{4}+\epsilon} z^{3}+\frac{t^{4}}{t^{3}+t^{4}+\epsilon} z^{4}\right] .
\end{aligned}
$$

Let

$$
\begin{aligned}
& s^{1}=t^{1}, s^{2}=t^{2}-\epsilon, s^{3}=t^{3}+t^{4}+\epsilon, \\
& \alpha^{2}=\frac{\epsilon}{s^{3}}, \alpha^{3}=\frac{t^{3}}{s^{3}}, \alpha^{4}=\frac{t^{4}}{s^{3}}, \\
& y=\alpha^{2} z^{2}+\alpha^{3} z^{3}+\alpha^{4} z^{4} .
\end{aligned}
$$

We conclude that dim $\operatorname{span}\left\{z^{1}, z^{2}, y\right\}=2$ so $\operatorname{span}\left\{z^{1}, z^{2}, y\right\}=\left(\mathbb{R}^{3}\right)_{b}$ for some $b \in \mathbb{R}^{3} \backslash\{0\}$. Proceeding as in the proof of Theorem 3.2, we find $\omega^{1} \in W_{0}^{1, \infty}\left(\Omega ; \mathbb{R}^{3}\right)$ satisfying

1. $\omega^{1}(x)=u(x) b$ for a.e. $x \in \Omega$, where $u \in W_{0}^{1, \infty}\left(\mathbb{R}^{3} ; \mathbb{R}\right)$ is a piecewise affine function satisfying a suitable gradient problem

2. $\Omega=\left\{x \in \mathbb{R}^{3} \mid u(x)>0\right\}$

3. $\operatorname{curl} \omega^{1}(x) \in\left\{z^{1}, z^{2}, y\right\}$, for a.e. $x \in \Omega$.

It can be shown that $\Omega$ is bounded and open (cf. Lemma 2.11 of [10]).

Step 2. As $\omega^{1}$ is piecewise affine, we can define the bounded, open set

$$
\Omega_{y}=\left\{x \in \Omega: \operatorname{curl} \omega^{1}(x)=y\right\} .
$$

Now, observing that $\left\{z^{2}-y, z^{3}-y, z^{4}-y\right\}$ and $\left\{\alpha^{2}, \alpha^{3}, \alpha^{4}\right\}$ satisfy

$$
0=\alpha^{2}\left(z^{2}-y\right)+\alpha^{3}\left(z^{3}-y\right)+\alpha^{4}\left(z^{4}-y\right),
$$


we can find $\omega^{2} \in W_{0}^{1, \infty}\left(\Omega_{y} ; \mathbb{R}^{3}\right)$ such that

$$
\operatorname{curl} \omega^{2}(x) \in\left\{z^{2}-y, z^{3}-y, z^{4}-y\right\} \text {, for a.e. } x \in \Omega_{y} .
$$

Finally, we define

$$
\omega(x)=\left\{\begin{array}{cl}
\omega^{1}(x) & \text { if } x \in \Omega \backslash \Omega_{y} \\
\omega^{1}(x)+\omega^{2}(x) & \text { if } x \in \Omega_{y} .
\end{array}\right.
$$

Evidently $\omega \in W_{0}^{1, \infty}\left(\Omega ; \mathbb{R}^{3}\right)$ and

$$
\operatorname{curl} \omega(x) \in\left\{z^{1}, z^{2}, z^{3}, z^{4}\right\} \subset E \text {, for a.e. } x \in \Omega .
$$

Necessary Condition. Let $u$ be a solution of (4.8). We show that there exists a subset $F \subseteq E$, such that $\operatorname{dim} \operatorname{span} F \geqslant 2$ and $0 \in \operatorname{ri}(\operatorname{co} F)$.

We begin by showing that $\operatorname{dim} \operatorname{span} E \geq 2$. Note that this was already proved in Theorem 4.5, but in a more complex way.

Suppose that $\operatorname{dim} \operatorname{span} E=1$. Then, there exist $\nu \in S^{2}$ and $\alpha=\alpha(x)$ such that

$$
\operatorname{curl} u(x)=\alpha(x) \nu, \quad \text { for a.e. } x \in \Omega .
$$

We extend $u$ by 0 to all of $\mathbb{R}^{3}$ and consider $B$ an open ball of radius $R$ such that $\bar{\Omega} \subset B$. Define $u_{\varepsilon}:=\rho_{\varepsilon} \star u$, where $\rho_{\varepsilon}$ is the standard mollifier. Let $\Omega_{\varepsilon}$ be such that $\operatorname{supp} u_{\varepsilon} \subset \Omega_{\varepsilon}$ and consider $\varepsilon_{0}>0$ small enough so that $\bar{\Omega}_{\varepsilon} \subset B$, for all $0<\varepsilon<\varepsilon_{0}$.

Notice that

$$
\operatorname{curl} u_{\varepsilon}(x)=\left(\rho_{\varepsilon} \star \alpha\right)(x) \nu=: \alpha_{\varepsilon}(x) \nu, \quad \text { for a.e. } x \in \Omega \text {. }
$$

We define

$$
\Phi(x):=-\int_{0}^{2 R}\langle u(x+t \nu) ; \nu\rangle d t
$$

and

$$
\Phi_{\varepsilon}(x):=-\int_{0}^{2 R}\left\langle u_{\varepsilon}(x+t \nu) ; \nu\right\rangle d t .
$$

It is easy to see that $\Phi_{\varepsilon}=\rho_{\varepsilon} \star \Phi$. We will now show that grad $\Phi_{\varepsilon}=u_{\varepsilon}$. By the usual identification of the vectors curl $u_{\varepsilon}$ and $\nu$ with $3 \times 3$ antisymmetric matrices, condition (4.10) may be written as

$$
\sum_{j=1}^{3} \alpha_{\varepsilon}^{i j} \nu^{j}=0, \quad i=1,2,3
$$


where $\left(\operatorname{curl} u_{\varepsilon}\right)^{i j}=\alpha_{\varepsilon}^{i j}$. Thus, for $i=1,2,3$, we have

$$
\begin{aligned}
\frac{\partial}{\partial x_{i}} \Phi_{\varepsilon}(x) & =-\int_{0}^{2 R} \sum_{j=1}^{3} \frac{\partial}{\partial x_{i}} u_{\varepsilon}^{j}(x+t \nu) \nu^{j} d t \\
& =-\int_{0}^{2 R} \sum_{j=1}^{3}\left(\frac{\partial u_{\varepsilon}^{i}}{\partial x_{j}}(x+t \nu)+\alpha_{\varepsilon}^{i j}\right) \nu^{j} d t \\
& =-\int_{0}^{2 R}\left\langle\nabla u_{\varepsilon}^{i}(x+t \nu) ; \nu\right\rangle d t \\
& =-\int_{0}^{2 R} \frac{d}{d t}\left[u_{\varepsilon}^{i}(x+t \nu)\right] d t=u_{\varepsilon}^{i}(x) .
\end{aligned}
$$

As $\operatorname{grad} \Phi_{\varepsilon}=u_{\varepsilon}$, it follows that curl $u_{\varepsilon}=0$. On the other hand,

$$
\operatorname{grad} \Phi_{\varepsilon}=\rho_{\varepsilon} \star \operatorname{grad} \Phi=\rho_{\varepsilon} \star u
$$

so we conclude that $u=\operatorname{grad} \Phi$, a.e. in $\Omega$. Hence $\operatorname{curl} u=0$, a.e. in $\Omega$, which contradicts our assumption that $0 \notin E$. Therefore, $\operatorname{dim} \operatorname{span} E \geq 2$.

If $\operatorname{dim} \operatorname{span} E=2$ we choose $F=E$. By Theorem 4.13 it follows that

$$
0 \in \operatorname{ri}(\operatorname{co} F)=\operatorname{ri}(\operatorname{co} E)
$$

If $\operatorname{dim} \operatorname{span} E=3$ we set $F=\operatorname{curl} u\left(\Omega^{\prime}\right)$ where $\Omega^{\prime} \subseteq \Omega$ is such that $\operatorname{curl} u(x) \in E$ for all $x \in \Omega^{\prime}$. The same argument used for span $E$ also allows us to conclude that $\operatorname{dim} \operatorname{span} F \geqslant 2$. Examining the proof of Theorem 3.6 (cf. [1], [2]) or Theorem 4.13 we deduce that $0 \in \operatorname{ri}(\operatorname{co} F)$.

This concludes the proof.

Acknowledgements. The research of A. C. Barroso and J. Matias was partially supported by the FCT, Portugal through the POCTI Programme. Part of this work was completed during visits of A. C. Barroso and J. Matias to EPFL, whose hospitality is gratefully acknowledged.

\section{References}

[1] Saugata Bandyopadhyay. Ph.D thesis. In preparation.

[2] Saugata Bandyopadhyay, Ana Cristina Barroso, Bernard Dacorogna, and José Matias. In preparation.

[3] Ana Cristina Barroso and José Matias. Necessary and sufficient conditions for existence of solutions of a variational problem involving the curl. Discrete Contin. Dyn. Syst., 12(1):97-114, 2005. 
[4] Alberto Bressan and Fabián Flores. On total differential inclusions. Rend. Sem. Mat. Univ. Padova, 92:9-16, 1994.

[5] Arrigo Cellina. On minima of a functional of the gradient: necessary conditions. Nonlinear Anal., 20(4):337-341, 1993.

[6] Arrigo Cellina. On minima of a functional of the gradient: sufficient conditions. Nonlinear Anal., 20(4):343-347, 1993.

[7] Bernard Dacorogna. Non convex problems of the calculus of variations and differential inclusions. To appear in Stationary Partial Differential equations, Handbook of Differential Equations. North-Holland Publishing Co., Amsterdam.

[8] Bernard Dacorogna. Existence and regularity of solutions of $d \omega=f$ with Dirichlet boundary conditions. In Nonlinear problems in mathematical physics and related topics, I, volume 1 of Int. Math. Ser. (N. Y.), pages 67-82. Kluwer/Plenum, New York, 2002.

[9] Bernard Dacorogna and Irene Fonseca. A-B quasiconvexity and implicit partial differential equations. Calc. Var. Partial Differential Equations, 14(2):115-149, 2002.

[10] Bernard Dacorogna and Paolo Marcellini. Implicit partial differential equations. Progress in Nonlinear Differential Equations and their Applications, 37. Birkhäuser Boston Inc., Boston, MA, 1999.

[11] Antonio DeSimone and Georg Dolzmann. Existence of minimizers for a variational problem in two-dimensional nonlinear magnetoelasticity. Arch. Rational Mech. Anal., 144(2):107-120, 1998.

[12] Gero Friesecke. A necessary and sufficient condition for nonattainment and formation of microstructure almost everywhere in scalar variational problems. Proc. Roy. Soc. Edinburgh Sect. A, 124(3):437-471, 1994.

[13] Werner Greub. Multilinear algebra. Springer-Verlag, New York, second edition, 1978. Universitext.

[14] Jean-Baptiste Hiriart-Urruty and Claude Lemaréchal. Fundamentals of convex analysis. Grundlehren Text Editions. Springer-Verlag, Berlin, 2001.

[15] Tadeusz Iwaniec. Nonlinear differential forms, volume 80 of Report. University of Jyväskylä Department of Mathematics, Jyväskylä, 1998. Lectures in Jyväskylä.

[16] Tadeusz Iwaniec and Gaven Martin. Geometric function theory and nonlinear analysis. Oxford Mathematical Monographs. The Clarendon Press Oxford University Press, New York, 2001. 
[17] Richard D. James and David Kinderlehrer. Frustration in ferromagnetic materials. Contin. Mech. Thermodyn., 2(3):215-239, 1990.

[18] R. Tyrrell Rockafellar. Convex analysis. Princeton Mathematical Series, No. 28. Princeton University Press, Princeton, N.J., 1970. 